\title{
Design and Evaluation of a Measurement Procedure to obtain the Electric Permittivity and the Magnetic Permeability
}

\author{
Diseño y evaluación del procedimiento de medición para obtener la permitividad eléctrica y la \\ permeabilidad magnética \\ Rittner I. L.*, Fano W. G. ${ }^{\dagger}$ \\ *Hamburg University of Technology \\ Am Schwarzenberg-Campus 1 - 21073 - Hamburg - Germany \\ i.rittneratuhh.de \\ $\dagger^{\dagger}$ Universidad de Buenos Aires, Departamento de Electrónica, Laboratorio de Radiación Electromagnética \\ Av. Paseo Colón 850 - C1063ACV - Buenos Aires - Argentina \\ gustavo.gf2005@gmail.com \\ Recibido: 05/05/18; Aceptado: 13/06/18
}

\begin{abstract}
An implementation of the transmission/reflection line method is presented to determine the intrinsic electromagnetic properties of unknown materials with a relative permeability not equal to one. A low-cost, easy to manufacture sample holder is realised using a coaxial transmission line with $\mathbf{N}$-type female connectors. The reflection and transmission coefficients are measured to simultaneously extract the electric permittivity and magnetic permeability. The classic Nicolson-Ross-Weir (NRW) extraction technique in frequency domain is presented such that the equations can be easily implemented with open-source tools such as GNU Octave. The shift in phase reference planes and connector calibration measurements are performed such that a lowcost vector network analyser (VNA) without de-embedding function can be used. The measurement procedure is valid from $2 \mathrm{MHz}$ to $6 \mathrm{GHz}$. The article also comments on the sample preparation especially the thickness of the material slab.
\end{abstract}

Keywords: transmission line measurements; parameter extraction; calibration.

Resumen- Se presenta una implementación del método de línea de transmisión / reflexión para determinar las propiedades electromagnéticas intrínsecas de materiales desconocidos con una permeabilidad relativa distinta de uno. Se construyó un portamuestras de bajo costo y fácil de fabricar utilizando una línea de transmisión coaxial con conectores hembra tipo $N$. Los coeficientes de reflexión y transmisión se miden para extraer simultáneamente la permitividad eléctrica y la permeabilidad magnética. La técnica clásica de extracción de Nicolson-Ross-Weir (NRW) en el dominio de la frecuencia se presenta de tal manera que las ecuaciones se pueden implementar fácilmente con herramientas de código libre como GNU Octave. El desplazamiento en los planos de referencia de fase y las mediciones de calibración del conector se realizan de tal manera que se puede usar un analizador vectorial de (VNA) de bajo costo sin las funciones incorporadas. El procedimiento de medición es válido desde $2 \mathrm{MHz}$ hasta $6 \mathbf{G H z}$. El artículo también comenta sobre la preparación de la muestra especialmente el espesor del bloque de material.

Palabras clave: mediciones en línea de transmisión; extracción de parámetros; calibración.

\section{INTRODUCTION}

In a technology dominated world there is a constant growth and an increasing diversity of applications to process and transmit microwave signals. Therefore it becomes increasingly interesting to design materials with desired behaviours in the presence of electromagnetic fields. Different techniques for the electromagnetic characterization of unknown materials have been developed. All of them have limitations based on the frequency range, the sample material to be used, the parameters to be obtained etc. In the following a brief overview of the procedures explained in detail in [1], [2] and [3] is given.

Resonant methods e.g. applying a cavity resonator can be used to measure the permittivity and permeability of very small samples of the material under test (MUT). The basic idea is that the material properties can be inferred from changes of the resonant frequency and the quality factor of the resonator. The results are obtained with very high accuracy but are limited to a narrow frequency band. With nonresonant methods the electromagnetic properties of materials are deduced from their impedance and wave velocities within the material. Reflection methods such as the openended coaxial probe method collect the reflected energy after it is directed toward a material. Therefore only the electric permittivity $\epsilon_{R}$ may be determined. The open-ended coaxial probe method is a non-destructive testing method usually used for biological specimens as the sample does not need to be machined to fit a sample holder. The electric permittivity $\epsilon_{R}$ may also be determined with the parallel plate capacitor method as used in [4]. Here the measurement device is constructed as a parallel plate capacitor, which is filled with the MUT alternating the capacitance. Voltage measurements are used to then calculate $\epsilon_{R}$. Transmission/reflection methods evaluate the reflected and transmitted energy directed toward a material. All types of transmission lines such as free-space, hollow metallic or dielectric waveguides may be used to carry the electromagnetic wave impinging on the material surface. The free-space measurement method can yield both 
parameters $\epsilon_{R}$ and $\mu_{R}$. It consists of an arrangement with two directive antennas facing each other connected to a VNA. The material is placed as a thin plate in the middle of the set-up. This method can be used to determine the electromagnetic parameters for a wide frequency range and allows high frequency measurements. The free-space measurements inherit the great disadvantage that the MUT has to be large and thin. With coaxial lines or metallic waveguides as sample holders smaller sample sizes may be used, which results in a destructive technique because the sample must be prepared to fit the sample holder. These transmissionline techniques allow a broadband characterization of the complex electromagnetic parameters $\epsilon_{R}$ and $\mu_{R}$ of unknown materials.

The measurement technique presented is designed for materials eventually being used for radio frequency absorbers. Therefore a wide-band method for the simultaneous determination of $\epsilon_{R}$ and $\mu_{R}$ for solid materials is needed. A two-port transmission-line technique is implemented using a coaxial transmission-line as sample holder. This coaxial transmission-line allows a transversal electromagnetic wave (TEM) to propagate such that the energy density of the TEM is tangential to the sample interface therefore increasing the measurement accuracy. The idea of the general procedure is to measure the forward- and back-scattered energy of electromagnetic waves in the frequency range $2 \mathrm{MHz}-6 \mathrm{GHz}$. The measurements are realised with a VNA at its connector reference planes. The data is then post-processed yielding the relationship of the forward- and back-scattered energy $S_{11}$ and $S_{21}$ at the sample interfaces, from which the intrinsic electromagnetic parameters $\epsilon_{R}$ and $\mu_{R}$ may be calculated as functions of frequency. To extract the complex parameters $\epsilon_{R}=\epsilon_{R}^{\prime}-j \epsilon_{R}^{\prime \prime}$ and $\mu_{R}=\mu_{R}^{\prime}-j \mu_{R}^{\prime \prime}$ from the measured scattering parameters $S_{11}$ and $S_{21}$ the classic NRW method is used [5], [6]. Later developed techniques such as the NIST iterative or the new non-iterative extraction or conversion technique [3] assume $\mu_{R}=1$. This makes these techniques not suitable for the proposed application as the synthesized absorbing materials may have $\mu_{R} \neq 1$. The equations derived in the original papers are extend like in [7] and implemented in the post-processing with the opensource software GNU Octave, which is similar to MATLAB.

\section{THEORY}

First of all, a theoretical model of the wave propagation inside the sample holder has to be derived in order to understand the derivation of the Nicolson-Ross-Weir Method [5], [6]. The original notation as in the first paper by Nicolson and Ross [5] is used. The influence of the connectors and the length of the coaxial line on the wave propagation is modelled as a shift in reference plane explained in section II-B.

\section{A. TEM Wave Propagation in the Presence of a Sample}

Figure 1 shows a sample slab of thickness $d$ positioned in the middle of the sample holder designed as an air-filled coaxial transmission line of length $L_{L}$. The screw holes for the connectors are indicated by the four small circles on each side.

In the presence of a current flow and a potential difference between the inner and outer conductor the electric and the

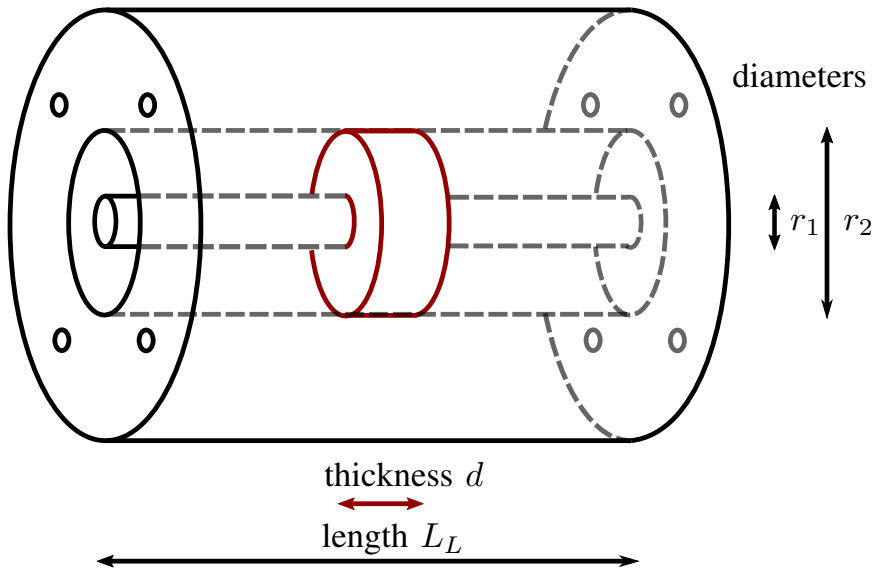

Fig. 1: Model of a sample holder as a coaxial TEM transmission line.

magnetic field are perpendicular over the entire coaxial transmission line. This enables the propagation of a TEM wave, which is represented by a voltage wave as indicated in figure 2. Under TEM conditions the forward-travelling voltage wave $V_{A_{i n c}}$ incides perpendicularly on the sample interface.

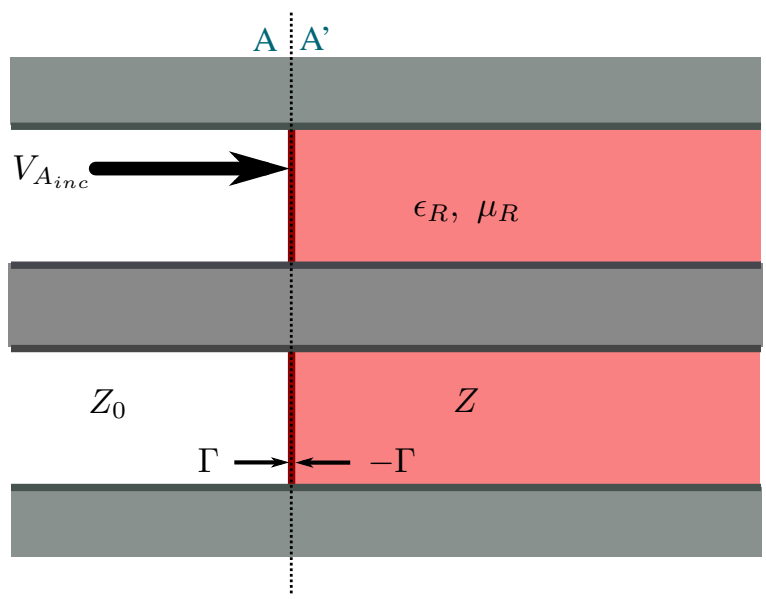

Fig. 2: Schematic of an air-to-sample interface A-A' for a semi-infinite sample inside a coaxial TEM transmission line.

At the air-to-sample interface denoted as A-A' in figure 2 a discontinuity occurs, when the characteristic impedance changes from $Z_{0}$ to $Z$. For the case of a semi-infinite sample part of the electromagnetic wave is reflected, while the other part is transmitted and propagates within the MUT. From field analysis of transmission lines [8] follows that the characteristic impedance depends on the factor $\sqrt{\frac{\epsilon_{0} \epsilon_{R}}{\mu_{0} \mu_{R}}}$, where $\epsilon_{0}$ and $\mu_{0}$ are the electric and magnetic constant and $Z_{0}=\sqrt{\frac{\mu_{0}}{\epsilon_{0}}}=377 \Omega$, yielding

$$
Z=\sqrt{\frac{\mu_{R}}{\epsilon_{R}}} Z_{0} .
$$

The propagation at the interface of a semi-infinite sample can be described by the voltage reflection coefficient 


$$
\Gamma=\frac{Z-Z_{0}}{Z+Z_{0}}=\frac{\sqrt{\frac{\mu_{R}}{\epsilon_{R}}}-1}{\sqrt{\frac{\mu_{R}}{\epsilon_{R}}}+1} .
$$

and the transmission coefficient $1+\Gamma$ [8]. Note that in the case of a wave propagating through the material and inciting on an interface with air its propagation is described by the reflection and transmission coefficients $-\Gamma$ and $1-\Gamma$.

Rewriting equation 2 yields the quotient $\frac{\mu_{R}}{\epsilon_{R}}$ as function $c_{1}$ of $\Gamma$ :

$$
\frac{\mu_{R}}{\epsilon_{R}}=\frac{(1+\Gamma)^{2}}{(1-\Gamma)^{2}}=c_{1}(\Gamma)
$$

At this point the propagation model has to be extended to include the wave propagation in the presence of a sample slab of finite thickness $d$ as depicted in figure 3 .

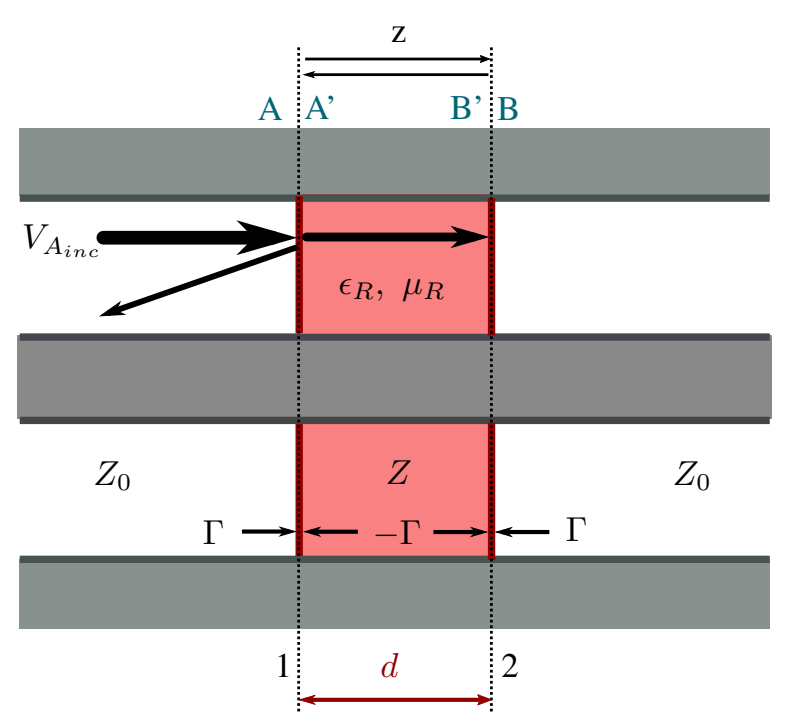

Fig. 3: Schematic of the two air-sample-interfaces when a sample of finite thickness $d$ is placed in the middle of a coaxial TEM transmission line.

In this case, the amplitude and phase change due to the propagation within the sample before the wave incides on the second interface has to be considered. In general, for a forward travelling wave in $+x$ direction the wave propagation is accounted for by the factor

$$
z(x)=e^{-\gamma x},
$$

where $\gamma=\alpha+j \beta=j k_{c}$ is the frequency-dependent, complex propagation constant with wave number $k_{c}$. The wavenumber may be represented as a function of the product $\sqrt{\mu_{R} \epsilon_{R}}[9]$ :

$$
k_{c}=\omega \sqrt{\mu \epsilon}=\frac{\omega}{c} \sqrt{\mu_{R} \epsilon_{R}},
$$

where $c=\left(\sqrt{\epsilon_{0} \mu_{0}}\right)^{-1}$ is the speed of light and $\omega$ the angular frequency.

The amplitude change and phase factor for a propagation distance of $x=d$ result in:

$$
z=e^{-j \frac{\omega}{c} \sqrt{\mu_{R} \epsilon_{R}} d}
$$

In the following this is denoted as the propagation factor $z$. With the propagtation factor $z$ the wave propagation within the sample can be described independent of the direction of the travelling wave as indicated in figures 3 and 4. Rewriting equation 6 enables the description of the product $\mu_{R} \epsilon_{R}$ as function of $z$ :

$$
\mu_{R} \epsilon_{R}=-\left(\frac{c}{\omega d} \ln \frac{1}{z}\right)^{2}=c_{2}(z)
$$

From $c_{1}(\Gamma)$ and $c_{2}(z)$ the electromagnetic parameters $\epsilon_{R}$ and $\mu_{R}$ may easily be determined. At this point $\Gamma$ and $z$ are needed as functions of the measured quantities $S_{11}^{\prime}$ and $S_{21}^{\prime}$ in order to calculate the permittivity and permeability of the material from the measurements being realised. The extension of the calibration surface from ports 1' and 2' to ports 1 and 2 is explained in section II-B. Therefore, first of all the relation between the scattering parameters $S_{11}$ and $S_{21}$ at ports 1 and 2 and the complex permittivity $\epsilon_{R}$ and permeability $\mu_{R}$ is derived in the following. The equations presented in [10] allow to rewrite equations 3 and 7 as functions of the cut-off wavelength $\lambda_{c}$ for a waveguide system as sample holder.

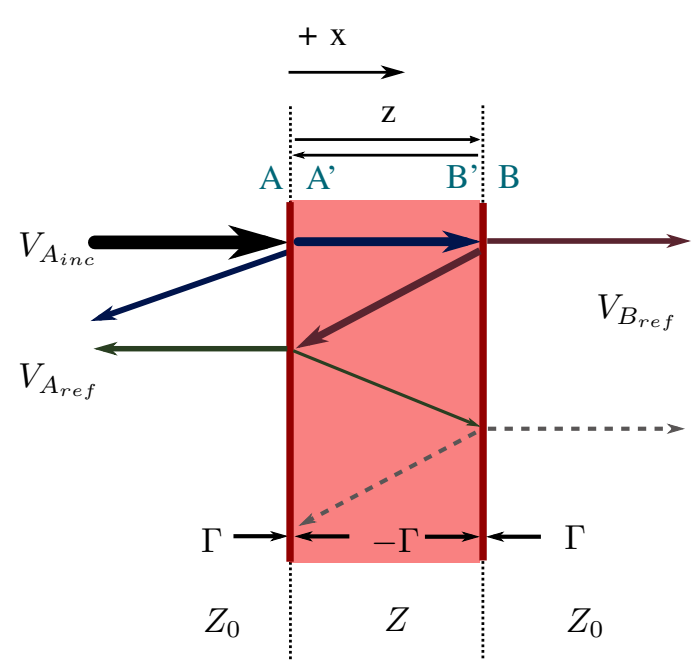

Fig. 4: Schematic of the incident signal $V_{A_{\text {inc }}}$, reflected signal $V_{A_{\text {ref }}}$ and transmitted signal $V_{B_{r e f}}$ with multiple reflections present inside the sample.

Figure 4 shows how a voltage wave, which incides at the interface $\mathrm{A}-\mathrm{A}$ ', propagates in the presence of a finite material sample. A complete bounce diagram indicating the time relations may be found in [11]. The incident voltage wave $V_{A_{i n c}}$ is both reflected and transmitted first at the airto-sample A-A' interface, later at the sample-to-air interface B'-B and then at the sample-to-air interface A'-A and so on. Therefore multiple reflections occur within the MUT. The reflected voltage waves at reference planes $\mathrm{A}$ and $\mathrm{B}, V_{A_{\text {ref }}}$ and $V_{B_{r e f}}$, consist of an infinite number of contributions with decreasing amplitude the more reflections within the sample have occurred. This propagation process may also be described by a signal flow graph. Figure 5 shows the signal flow graph presented in [5], from which equations 8 and 9 can be inferred.

The signal flow graph allows to relate the incident wave at reference plane A to the reflected waves at reference planes 


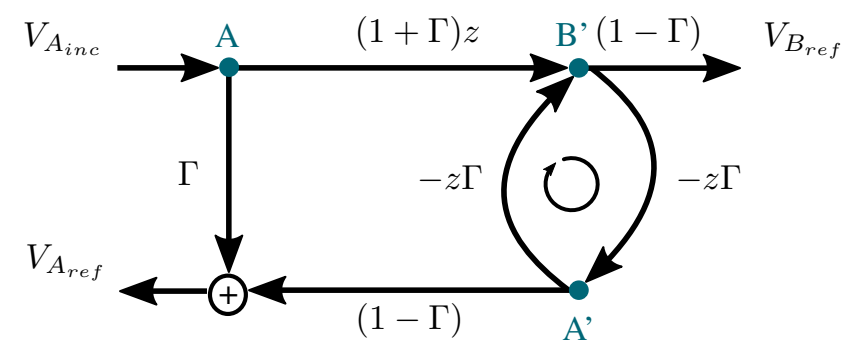

Fig. 5: Signal flow graph connecting the reference planes A, $\mathrm{A}^{\prime}$ and B'.

A and B, from which the scattering parameters $S_{11}$ and $S_{21}$ may be determined as functions of $z$ and $\Gamma$. The graph in figure 5 interrelates the reflections and transmissions of $V_{A_{\text {inc }}}$ at the air-to-sample interface with reference plane A, the sample-to-air interface with reference plane B' and the sample-to-air interface with reference plane A'. At A the impinging wave is partly reflected, which is represented by the factor $\Gamma$. The transmitted part also propagates through the finite sample of thickness $d$, which is described by the factor $(1+\Gamma) z$. At B' the reflection coefficient becomes $-\Gamma$ as indicated in figure 3 resulting in a factor of $1-\Gamma$ for the transmitted part. The reflected part at this sample-to-air interface again propagates through the sample represented by a total factor of $-z \Gamma$. When a fraction of $V_{A_{\text {inc }}}$ incides on the sample-to-air interface $A^{\prime}(1-\Gamma)$ is transmitted and the reflected part again propagates through the sample, which is described by the factor $-z \Gamma$. Multiple reflection occur between B' and A'.

a)

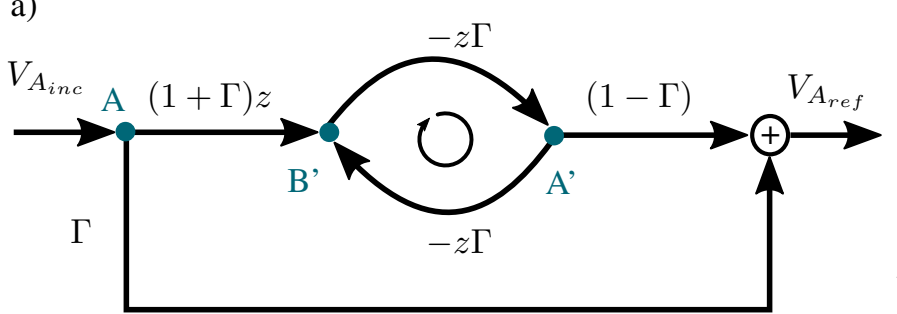

b)

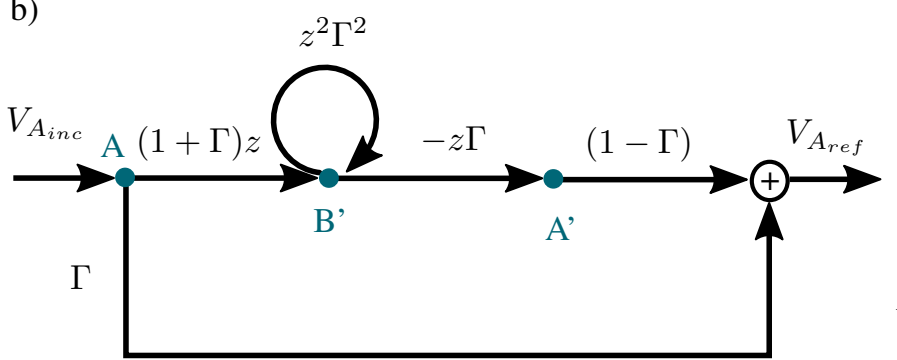

Fig. 6: Modified signal flow graph a) and simplification b) for the determination of the scattering parameter $S_{11}$.

Figure 6 shows the relation of $V_{A_{i n c}}$ and $V_{A_{\text {ref }}}$. From the version in figure $6 \mathrm{~b}$ ), which is simplified according to the rules in [8], $S_{11}$ can be determined as

$$
\begin{aligned}
S_{11} & =\frac{V_{A_{\text {ref }}}}{V_{A_{\text {inc }}}}=\frac{(1+\Gamma) z(-z \Gamma)(1-\Gamma)}{\left(1-\Gamma^{2} z^{2}\right)}+\Gamma \\
& =\frac{\left(1-z^{2}\right) \Gamma}{\left(1-\Gamma^{2} z^{2}\right)} .
\end{aligned}
$$

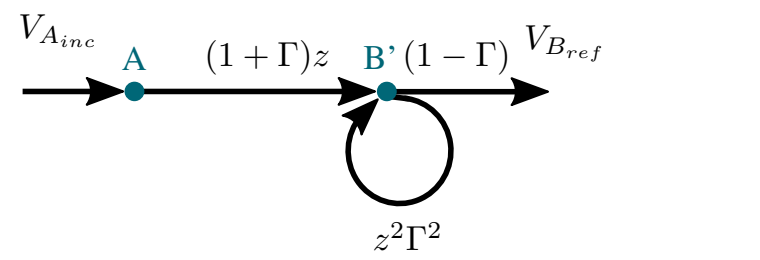

Fig. 7: Signal flow graph for the determination of the scattering parameter $S_{21}$.

The modified signal flow graph in figure 7 shows the relation of $V_{A_{\text {inc }}}$ and $V_{B_{\text {ref }}}$, which allows the determination of $S_{21}$ according to [8] as

$$
S_{21}=\frac{V_{B_{r e f}}}{V_{A_{\text {inc }}}}=\frac{(1+\Gamma) z(1-\Gamma)}{\left(1-\Gamma^{2} z^{2}\right)} .
$$

Nicolson and Ross in [5] combined equations 8 and 9 such that the system of equations decouples [7] resulting in explicit equations for the reflection coefficient $\Gamma$ and the propagation factor $z$ as functions of $S_{11}$ and $S_{21}$ :

$$
\begin{aligned}
\Gamma & =\frac{1-\left(S_{21}+S_{11}\right)\left(S_{21}-S_{11}\right)}{2 S_{11}} \\
& \pm \sqrt{\left(\frac{1-\left(S_{21}+S_{11}\right)\left(S_{21}-S_{11}\right)}{2 S_{11}}\right)^{2}-1}
\end{aligned}
$$

with the passivity condition of the material such that $|\Gamma| \leq$ 1 and

$$
z=\frac{S_{21}+S_{11}-\Gamma}{1-\left(S_{21}+S_{11}\right) \Gamma} .
$$

Equations 3 and 7 can then be written as functions of the scattering parameters enabling the determination of the intrinsic electromagnetic parameters as

$$
\epsilon_{R}=\sqrt{\frac{c_{2}\left(S_{11}, S_{21}\right)}{c_{1}\left(S_{11}, S_{21}\right)}}
$$

and

$$
\mu_{R}=\sqrt{c_{1}\left(S_{11}, S_{21}\right) c_{2}\left(S_{11}, S_{21}\right)} .
$$

\section{B. Shift of the Reference Planes to the Sample Surfaces}

In order to calculate $S_{11}$ and $S_{21}$ from the measured quantities $S_{11}^{\prime}$ and $S_{21}^{\prime}$ the VNA reference planes at port 1' and 2' have to be shifted to the sample surface with ports 1 and 2 . Figure 8 illustrates the different calibration reference planes. For the coaxial sample holder classic transmission line theory can be applied to shift the phase reference planes. The sample holder may be modelled by the two line model shown in figure 8 , where the two lines represent the 
inner and outer conductor of the coaxial transmission line. Since the sample holder may be approximated as a lossless transmission line, the shift for a forward travelling wave is done with the correcting phase factor

$$
e^{-j \beta L}=e^{-j \frac{2 \pi}{\lambda_{0}} L},
$$

where $\lambda_{0} \cong \frac{c_{0}}{f}$ is the wavelength in air and $\beta=\frac{2 \pi}{\lambda_{0}}$. $L$ denotes the equivalent electrical length of an air-filled transmission line, by which the phase reference plane is extended. Note that equation 14 is a simplification of the general propagation factor represented in equation 4. For a symmetrically placed sample the scattering parameters at ports 1 and 2 become

$$
S_{11}=S_{11}^{\prime} e^{j 2 \beta L}=S_{11}^{\prime} e^{j \frac{4 \pi}{\lambda_{0}} L}
$$

and

$$
S_{21}=S_{21}^{\prime} e^{j 2 \beta L}=S_{21}^{\prime} e^{j \frac{4 \pi}{\lambda_{0}} L}[8] .
$$

The scattering parameters relate the amplitudes of two waves, a forward and a backward travelling one. That is why a factor of 2 occurs when the reference plane is shifted by the length of $L$. As shown in figure 8 the forward travelling wave propagates over a transmission line of length $L$ until it incides on the sample surface. When the electrical length of the connector $L_{C}$ is included $L$ results in $L=\frac{1}{2}\left(L_{L}-d\right)+L_{C}$. [7] provides equations for any reference plane position, therefore applicable when the sample is not exactly placed in the middle.

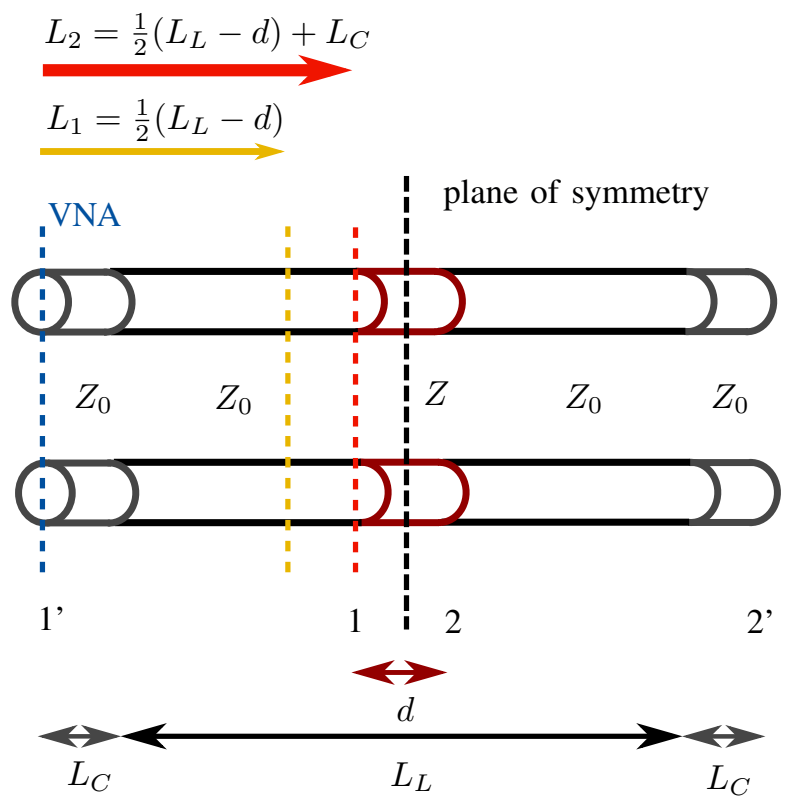

Fig. 8: Transmission line model with different reference planes corresponding to three phase factor arguments.

The results in figure 9 demonstrate how important it is to include $L_{C}$ in the reference plane shift. The blue, dotted line shows the results for $L=0$ meaning that no phase adjustment of the measured scattering parameters has been

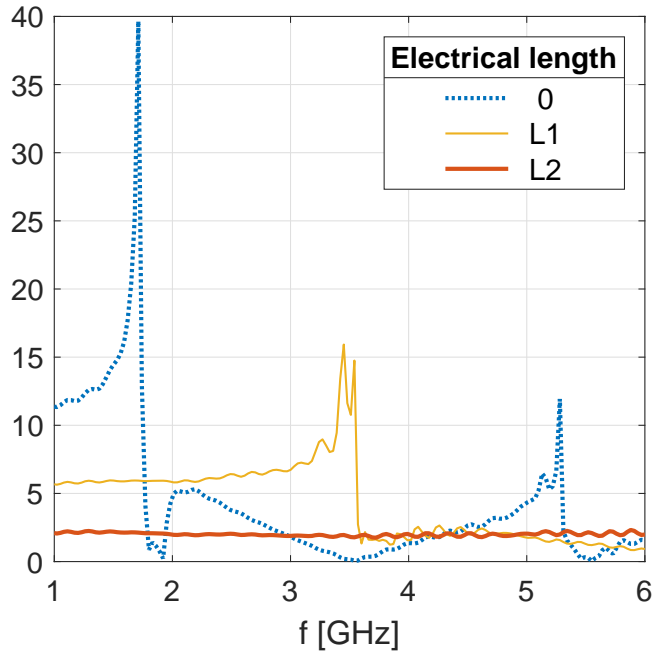

Fig. 9: Calculation results for the real part of $\epsilon_{R}$ for $L=0$, $L=L_{1}$ and $L=L_{2}$ corresponding to the three reference planes of figure 8 .

done. The results improve when $L=L_{1}=\frac{1}{2}\left(L_{L}-d\right)$ is chosen as indicated by the yellow curve. When additionally the wave propagation in the Teflon-filled connector is calibrated, which results in $L=L_{2}=\frac{1}{2}\left(L_{L}-d\right)+L_{C}$, the permittivity approaches the known value $\epsilon_{R}=2,1$ [12] independent of frequency.

The Teflon-filled connector is modelled as a transmission line with characteristic impedance $Z_{0}$ for the determination of the equivalent electrical length $L_{C}$. As electromagnetic waves propagate differently in air and Teflon, $L_{C}$ is not equal to the physical length of the real Teflon-filled connector. The employed transmission line model assumes a wave propagation in air like in the coaxial sample holder, in order to apply the phase correction with equations 15 and 16. $L_{C}$ may then be determined with the help of an impedance transformation. The empty sample holder is terminated and as the voltage amplitude for a mismatched line varies with position, $L_{C}$ can be inferred from input impedance calculations.

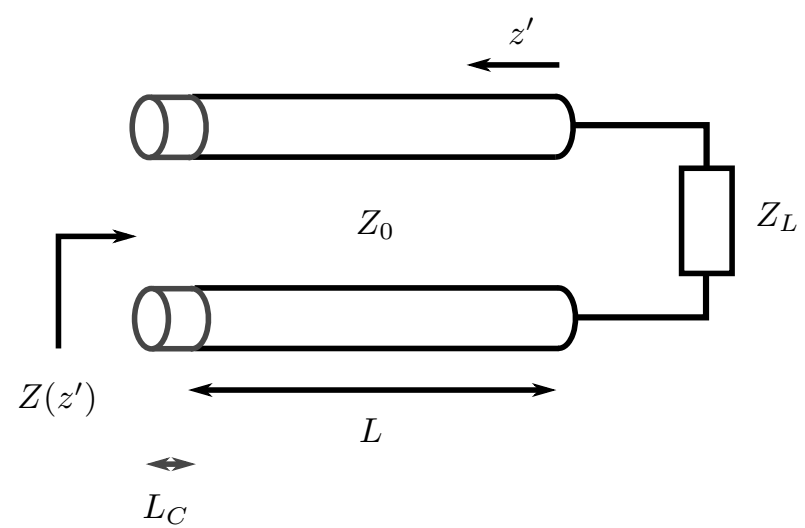

Fig. 10: Transmission line model for the connector calibration measurements.

Figure 10 shows a transmission line model of the termi- 
nated sample holder with one connector screwed on. For this lossless transmission line terminated with $Z_{L}$ the input impedance as function of $z^{\prime}$ becomes

$$
Z\left(z^{\prime}\right)=Z_{0} \frac{Z_{L}+j Z_{0} \tan \left(\beta z^{\prime}\right)}{Z_{0}+j Z_{L} \tan \left(\beta z^{\prime}\right)}[8] .
$$

This result is referred to as the transmission line impedance equation. If the transmission line is shortcircuited with $Z_{L}=0$ equation 17 simplifies to

$$
Z\left(z^{\prime}\right)=j Z_{0} \tan \left(\beta z^{\prime}\right)[8] .
$$

At $z^{\prime}=L_{L}+L_{C}$ the impedance of a short-circuited line can be calculated from frequency dependent reflection coefficient measurements using equation 2. The absolute value of $Z\left(L_{L}+L_{C}\right)$ converges to infinity, if $\beta z^{\prime}=$ $\beta\left(L_{L}+L_{C}\right)=n \frac{\pi}{2}$ with $n=1,2,3, \ldots$ Therefore for the first frequency $f_{1}$, for which $\left|Z\left(f_{1}\right)\right| \rightarrow \infty$, holds that $\beta\left(L_{L}+L_{C}\right)=\frac{2 \pi}{c_{0}} f_{1}\left(L_{L}+L_{C}\right)=\frac{\pi}{2}$ and

$$
L_{C}=\frac{c_{0}}{4 f_{1}}-L_{L} .
$$

For the coaxial air-filled line the electrical length coincides with the physical length $L_{L}$.

The VNA is first calibrated at the connector calibration planes. In case the VNA used does not have a de-embedding function as mentioned in [3], the extension of the reference planes and the connector and line calibration have to be implemented in the post-processing. Therefore the calibration procedure explained above provides a simple method to significantly upgrade a low-cost VNA.

\section{DESIGN}

\section{A. Sample Holder}

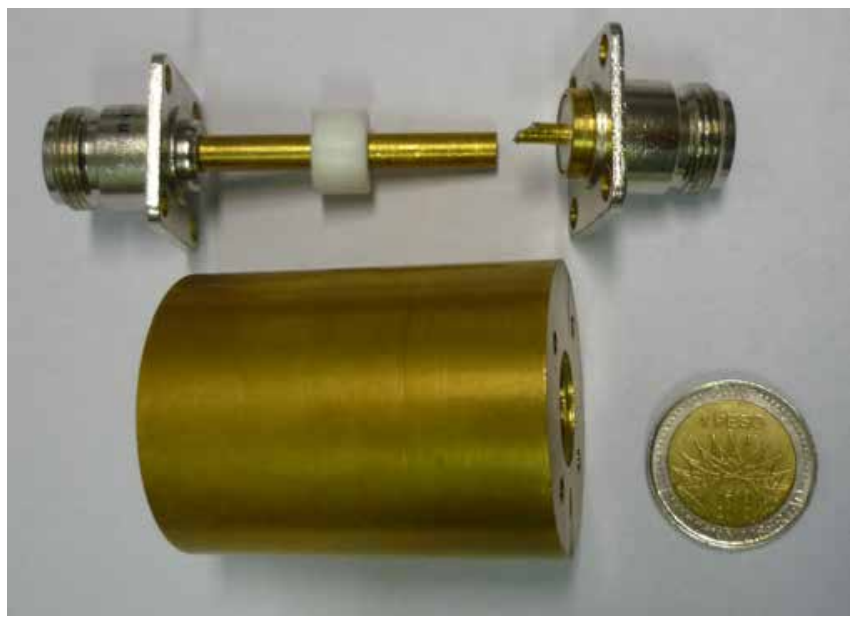

Fig. 11: Sample holder with the inner and outer cylindrical conductor, a Teflon sample with $d=8 \mathrm{~mm}$ and two connectors.

The sample holder is designed as a coaxial transmission line of length $L_{L}=50.4 \mathrm{~mm}$. The conductors of the coaxial line are made out of a solid bronze cylinder. Bronze is chosen because it provides high conductivity and may be machined easily. The geometry is designed to match the $50 \Omega$ Amphenol $\mathrm{N}$ type female connectors. Figure 11 shows the parts of the coaxial sample holder. The diameters $r_{1}$ and $r_{2}$ of the two cylindrical conductors indicated in figure 1 are determined according to:

$$
Z_{0}=\sqrt{\frac{\mu_{0} \mu_{R}}{\epsilon_{0} \epsilon_{R}}} \frac{1}{2 \pi} \ln \frac{r_{2}}{r_{1}}[8] .
$$

For a coaxial transmission line with air as dielectric the geometry dependent characteristic impedance $Z_{0}$ yields

$$
Z_{0} \approx 60 \ln \frac{r_{1}}{r_{2}} \Omega
$$

$r_{1}=5,4 \mathrm{~mm}$ and $r_{2}=12,5 \mathrm{~mm}$ are chosen, which yields $Z_{0} \approx 50,3 \Omega$, which has been confirmed by impedance measurements. Since the diameters of the $50 \Omega$ Teflon-filled connectors do not coincide with the chosen values for $r_{1}$ and $r_{2}$, adjustments have to be done. Figure 12 shows the bronze ring and the solder used to adjust the connectors. Note that inaccuracies in the manufacturing process of the ring could produce discontinuities at the connector transitions.

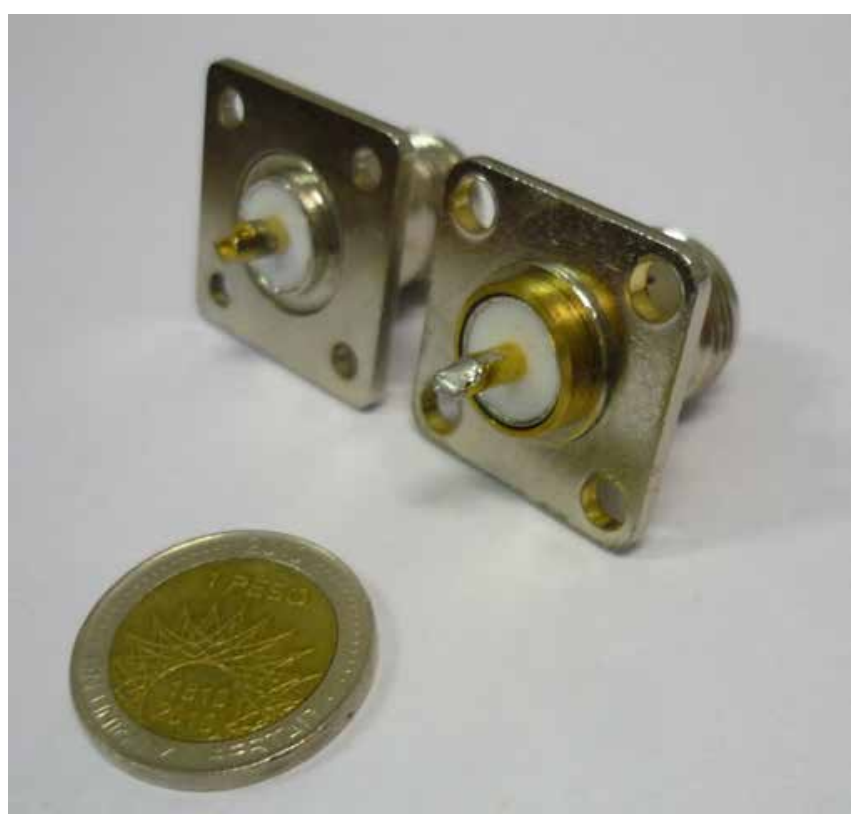

Fig. 12: Adjusted connector and original reference connector.

The connector calibration measurements as explained in section II-B are realised with the equipment shown in figure 13. The sample holder was manufactured in the mechanic's workshop of the Facultad de Ingeniería de la Universidad de Buenos Aires with a tolerance of $\pm 0.05 \mathrm{~mm}$.

\section{B. Sample Preparation}

To evaluate the measurement procedure different Teflon slab thickness as shown in figure 14 have been investigated. Also the results of three slabs of an unknown nickel ferrite (FNI) have been calculated. The samples have been manufactured with a tolerance of $\pm 0.1 \mathrm{~mm}$. Figures $17 \mathrm{~b}$ and $17 \mathrm{a}$ show that the results of the measurement procedure used depend on the sample slab thickness. However, this should not be the case, as the parameters are intrinsic. Therefore, criteria for suitable slab thicknesses need to be established investigating the behaviour of the known Teflon slabs. [3] 


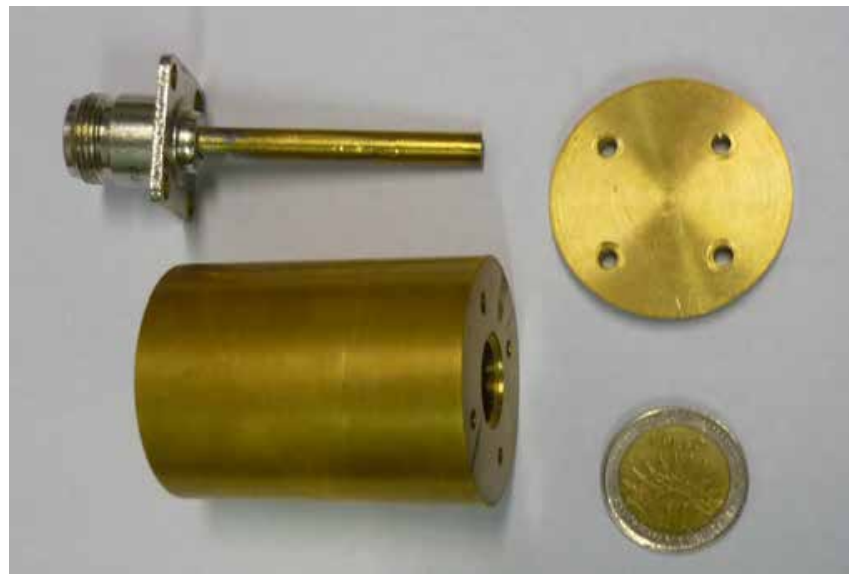

Fig. 13: Utensils used to measure the transmission line in short-circuit.

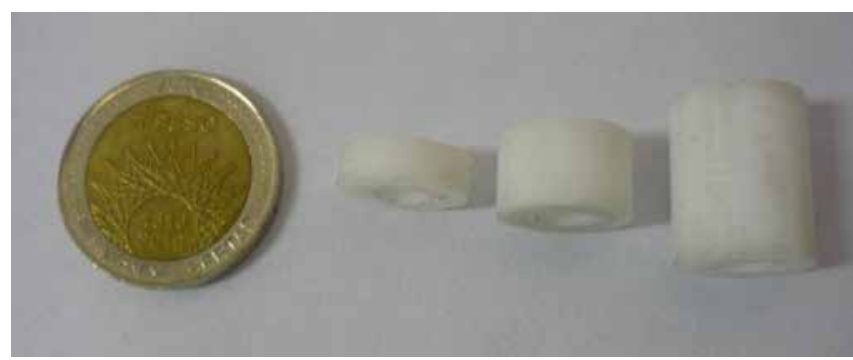

Fig. 14: Teflon slabs of thicknesses $d=4 \mathrm{~mm}, d=8 \mathrm{~mm}$ and $d=16 \mathrm{~mm}$.

proposes that $d \approx \frac{\lambda_{g}}{4}$ should be chosen, where $\lambda_{g}$ is the wavelength in the sample. For $f_{\max }=6 \mathrm{GHz}$

$$
\lambda_{g_{\min }}=\frac{v}{f}=\frac{c_{0}}{f_{\max } \sqrt{\epsilon_{R}}}
$$

can be determined. For Teflon $\frac{\lambda_{g_{\min }}}{4}=8,6 \mathrm{~mm}$ and the sample with $d=8 \mathrm{~mm}$ should yield the best results. The error analysis of figures $17 \mathrm{~b}$ and $17 \mathrm{a}$ proves that this is indeed the case. Therefore also for FNI a sample with $d \approx \frac{\lambda_{g_{\min }}}{4}$ should be chosen. To determine $\lambda_{g}$ of unknown samples such as FNI a posteriori knowledge of $\epsilon_{R}$ has to be used. The results of figure $15 \mathrm{~b}$ justify to set $\epsilon_{R}=3-5$ resulting $\frac{\lambda_{g_{\min }}}{4}=7,2-5,6 \mathrm{~mm}$. The imaginary part of the permittivity can be determined to be negligible. It follows that the FNI sample with $d=6,5$ yields the most accurate results.

For both MUT, Teflon and FNI, a divergence phenomenon occurs for the largest sample thickness $d$. As explained in [7] the classic NRW method diverges, when the sample thickness approaches multiples of $\frac{\lambda_{g}}{2}$. Using equation 22 results in $\frac{\lambda_{g}}{2} \approx 17,3 \mathrm{~mm}$ for Teflon and $\frac{\lambda_{g}}{2} \approx 14,4-12,5 \mathrm{~mm}$ for FNI, when a permittivity $\epsilon_{R} \approx 3-4$ is a posteriori assumed. This explains the divergence of the Teflon sample with $d=16 \mathrm{~mm}$ and the FNI sample with $d=15 \mathrm{~mm}$. [7] proposes an iterative procedure based on the NRW method to determine the complex permittivity independent on $d$. It is only suitable for nonmagnetic materials as $\mu_{R}$ is set to 1 .

For the symmetric placement of the samples the instrument shown in figure 16 has been manufactured. The

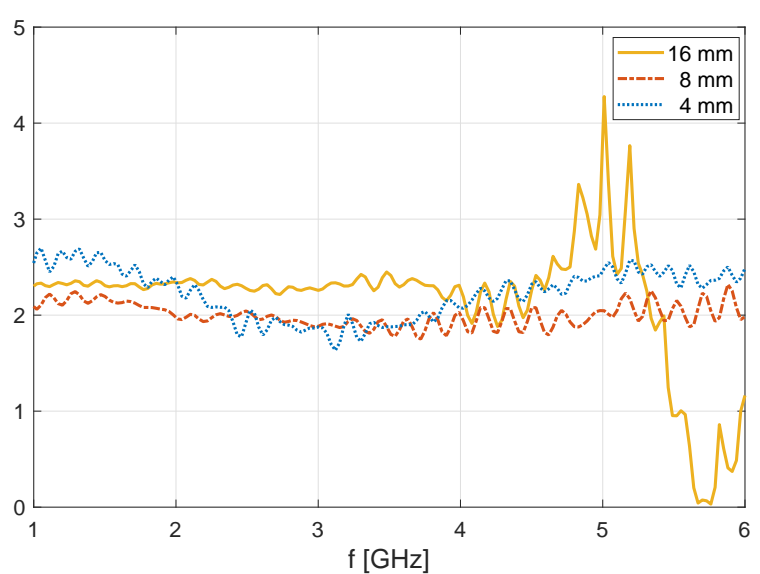

(a) Teflon.

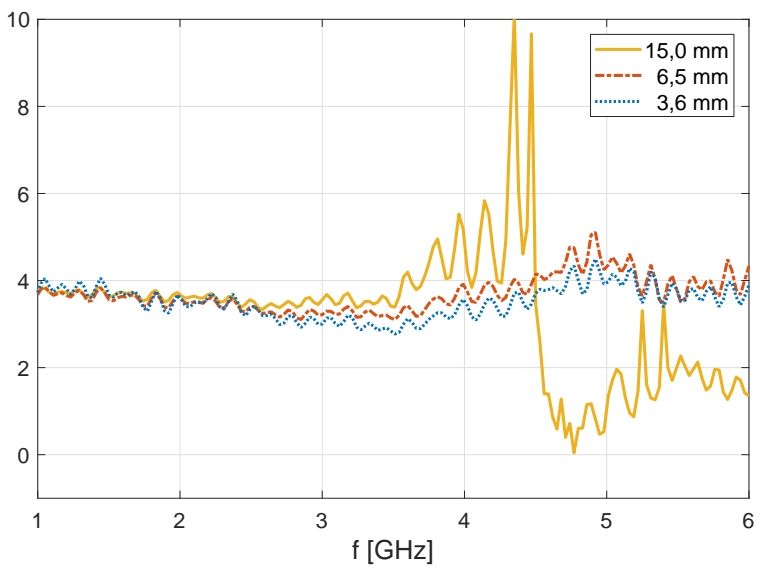

(b) FNI.

Fig. 15: Real part of $\epsilon_{R}$ for different slab thicknesses.

cylindrical element is hollow and has a diameter in between the diameters $r_{1}$ and $r_{2}$.

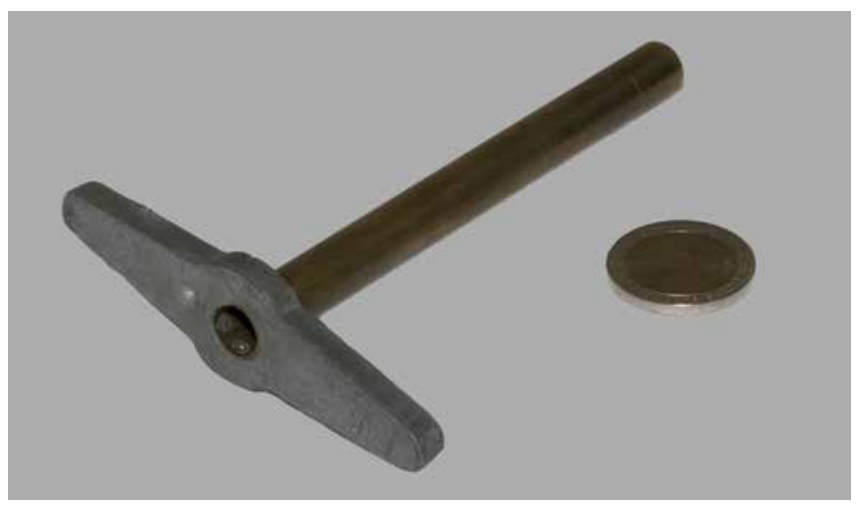

Fig. 16: Instrument to place the samples.

\section{RESULTS}

The accuracy of the proposed measurement procedure is assessed with the help of a well characterized material. Therefore, the GNU Octave [13] calculation results of a known Teflon sample are analysed. As discussed in section III-B slab thickness $d=8 \mathrm{~mm}$ is used. The complex permittivity and permeability are calculated from the scattering parameters measured with an Agilent Field Fox N9923A 


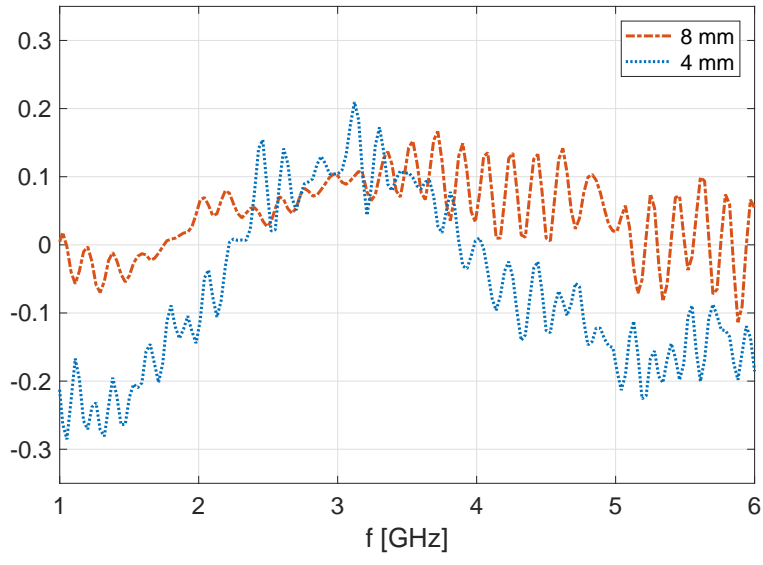

(a) Relative error of $\left|\epsilon_{R}\right|$.

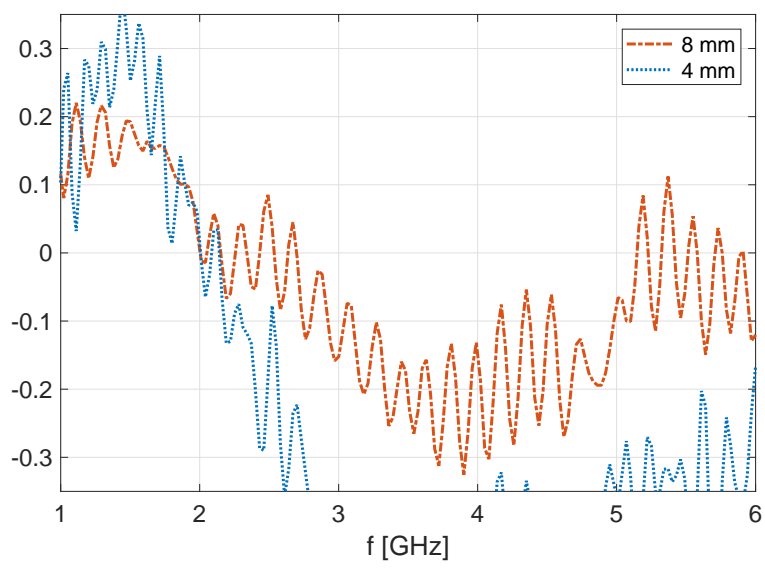

(b) Relative error of $\left|\mu_{R}\right|$.

Fig. 17: Relative error for Teflon with $d=4 \mathrm{~mm}$ and $d=$ $8 \mathrm{~mm}$.

vector network analyser, which works up to $6 \mathrm{GHz}$ and is calibrated by a standard two port full calibration with the provided $\mathrm{T}$ tool. The preliminary results are presented in the frequency range $1-6 \mathrm{GHz}$. Figures $18 \mathrm{a}$ and $18 \mathrm{~b}$ show the most accurate results for the appropriate slab thickness. Figures 17a and 17b illustrates that indeed $d=8 \mathrm{~mm}$ yields the smallest deviation from the known parameters for the dielectric. It can be seen that the accuracy of the measurement procedure can be characterized by a relative error below \pm 0.17 for $\epsilon_{R}$ and below \pm 0.33 for $\mu_{R}$.

The results presented in figures 18a and 18b show small and overall fluctuations, which may partly be attributed to the NRW algorithm itself. Previously published results of the complex permittivity and permeability determined according to the NRW method show similar fluctuations [7]. Further measurement uncertainties may be due to sources of possible impedance discontinuities for example at the transition to the connectors as mentioned in section III-A. Future designs of the sample holder focus on a smooth transition between the coaxial line and the connectors. Air gaps between the sample and the coaxial line are mentioned in [3] as a possible reason for measurement deviations. Also the calibration of the transmission line itself may be revised.

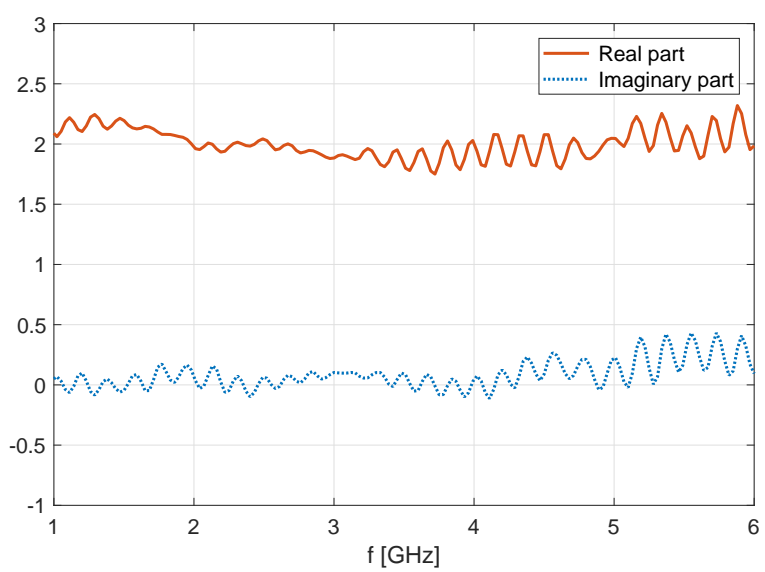

(a) $\epsilon_{R}$.

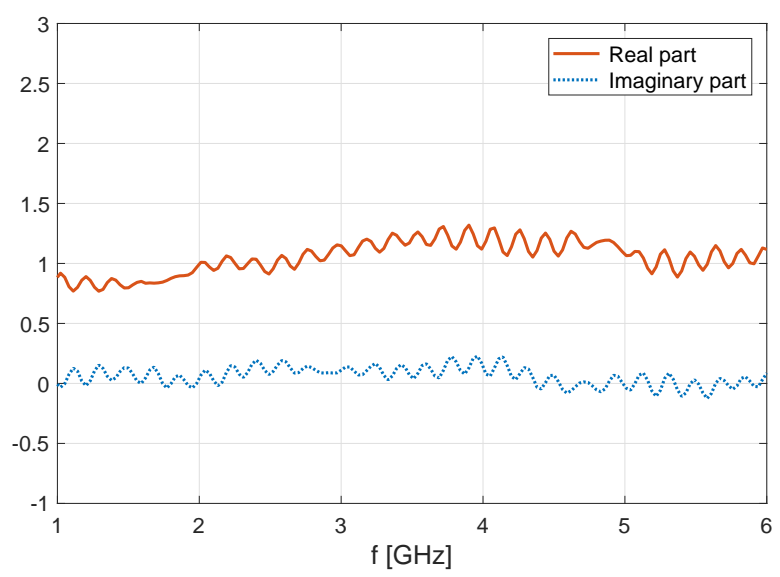

(b) $\mu_{R}$.

Fig. 18: Results for Teflon with $d=8 \mathrm{~mm}$.

\section{Conclusions}

In the article a signal flow graph approach to derive the classic NRW equations is explained in detail. Equations are presented to shift the phase reference plane of the measured scattering parameters in order to calculate the complex permittivity and permeability. An approach to determine the preferable sample slab thickness is derived. Finally measurements of the known dielectric Teflon show that the classic NRW method may be easily implemented up to $6 \mathrm{GHz}$ with a coaxial transmission line made of bronze with $\mathrm{N}$ type female connectors. The line has been designed to yield a characteristic impedance of $Z_{0}=50 \Omega$. An GNU Octave script has been written to compute and plot the results of the electric permittivity and the magnetic permeability. This post-processing also includes connector and line calibrations. The results of $\epsilon_{R}$ and $\mu_{R}$ as functions of frequency show small fluctuations attributed to the NRW algorithm itself, which have been reported by other researchers. This problem will be discussed in future papers of the authors.

Future works also consist of the design and implementation of a new sample holder with the aim to reduce discontinuities at the transition from the coaxial transmission line to the $\mathrm{N}$ type female connectors. An error analysis will also be provided. Furthermore an alternative post-processing 
algorithm as presented in [10] may be implemented to yield accurate results independent of the slab thickness $d$.

\section{ACKNOWLEDGEMENTS}

The authors acknowledge the fruitful discussions with Silvia Jacobo and Carlos Herme, and the help of Eriel Fernandez Galvan and his team manufacturing and adjusting the sample holders. The work reported in this article was realised by the kind collaboration and assistance of Carlos Herme in preparing and measuring the samples. Finally the authors would like to especially thank Ramiro Alonso and Valentino Trainotti for their constant support and motivation.

\section{REFERENCES}

[1] L. F. Chen, C. K. Ong, C. P. Neo, V. V. Varadan, and V. K. Varadan, Microwave Electronics. Chichester, UK: John Wiley \& Sons, Ltd, 2004.

[2] L. Sandrolini, U. Reggiani, and M. Artioli, "Electric and magnetic characterization of materials," in Behaviour of Electromagnetic Waves in Different Media and Structures, A. Akdagli, Ed. InTech, 2011.

[3] K. C. Yaw, "Measurement of dielectric material properties: Application note." [Online]. Available: https://cdn.rohdeschwarz.com/pws/dl downloads/dl' application/00aps undefined/RAC0607-0019'1'5E.pdf

[4] W. G. Fano and V. Trainotti, Eds., Dielectric Properties of Soils, 2001.

[5] A. M. Nicolson and G. F. Ross, "Measurement of the intrinsic properties of materials by time-domain techniques," IEEE Transactions on Instrumentation and Measurement, vol. IM-19, no. 4, pp. 377-382, 1970.

[6] W. B. Weir, "Automatic measurement of complex dielectric constant and permeability at microwave frequencies," Proceedings of the IEEE, vol. 62, no. 1, pp. 33-36, 1974.

[7] J. Baker-Jarvis, E. J. Vanzura, and W. A. Kissick, "Improved technique for determining complex permittivity with the transmission /reflection method," IEEE Transaction on Microwave Theory and Techniques, vol. 38, no. 8, August 1990.

[8] D. M. Pozar, Microwave engineering, 4th ed. New Delhi: Wiley India, 2012.

[9] D. K. Cheng and E. Morales Peake, Fundamentos de electromagnetismo para ingeniería. México: Addison-Wesley, 1998 [reimpresión].

[10] A. L. de Paula, M. C. Rezende, and J. J. Barroso, Eds., Modified Nicolson-Ross-Weir (NRW) method to retrieve the constitutive parameters of low-loss materials, 2011.

[11] C. C. Courtney, "Time-domain measurement of the electromagnetic properties of materials," IEEE Transaction on Microwave Theory and Techniques, vol. 46, no. 5, May 1998.

[12] A. R. von Hippel, Dielectric materials and applications, new ed. ed., ser. Artech House microwave library. Boston: Artech House, 1995.

[13] Eaton, J. W. et al., "Gnu octave." [Online]. Available: https://www.gnu.org/software/octave/ 